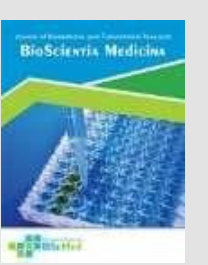

Bioscientia Medicina: Journal of Biomedicine \& Translational Research

Journal Homepage: www.bioscmed.com

\title{
Treatment of Systemic Lupus Erythematosus with Anti SSA and SSB Positive Pregnancies
}

\section{Robby Satria Putra ${ }^{*}$, Najirman ${ }^{1}$}

${ }^{1}$ Faculty of Medicine, Universitas Andalas, Padang, Indonesia

\section{A R T I C L E I N F O \\ Keywords: \\ Systemic lupus erythematosus \\ Pregnancy \\ Anti Ro/SSA \\ Anti La/SSB \\ *Corresponding author: \\ Robby Satria Putra \\ E-mail address: \\ drrobirini@gmail.com}

All authors have reviewed and approved the final version of the manuscript.

https://doi.org/10.37275/bsm.v6i3.470

\begin{abstract}
A B S T R A C T
Systemic lupus erythematosus (SLE) is autoimmune disease that results in inflammation in various organ systems, frequent recurrences in the clinical course characterized by the production of antibodies against core antigens. Pregnancy will trigger flares. Increased intravascular volume in pregnancy can worsen abnormal heart and kidney function. Increasing the glomerular filtration rate by $50 \%$ exacerbated previously stable proteinuria. Pregnancy-induced hypercoagulability increases the risk of thrombosis. Anti-Ro/SSA was found in $35 \%$ of SLE patients and anti-La / SSB was found in 15\% of SLE patients. The presence of Ro and La antibodies puts the fetus at a risk of developing neonatal lupus erythematosus (NLE) $6-13 \%$ and can reach up to $25 \%$ if the mother has previously given birth to a child with NLE. Neonatal lupus erythematosus including congenital heart block or cutaneous lupus. Congenital heart block (CHB) is a very serious complication. The American College of Rheumatology (ACR) recommends serial fetal echocardiography from 16 weeks of gestation continuing through to 26 weeks. Pregnancies with grade I and II CHB are treated with oral dexamethasone $4 \mathrm{mg}$ daily because fluorinated glucocorticoids such as dexamethasone and betamethasone can cross the placenta, however, this therapy cannot be given in third-degree heart block. Early diagnosis and therapy will reduce the risk of congenital heart block in neonates after birth.
\end{abstract}

\section{Introduction}

Systemic lupus erythematosus is an autoimmune disease that causes inflammation in various organ systems, often relapses in its clinical course characterized by the production of antibodies against nuclear antigens. Involvement of several organs in SLE is caused by the deposition of immune complexes in these organs. The etiopathogenesis is still unclear, genetic, environmental, hormonal factors play a role in the development of SLE. 1

Pregnancy is at risk for flares in SLE disease. Physiological and hormonal changes during pregnancy trigger lupus activity, as well as an increased inflammatory response by the SLE causing significant complications during pregnancy. Inflammation that occurs in the uterus can damage the placenta, causing inadequate blood flow, nutrition, and oxygen to the fetus, resulting in intrauterine growth retardation. Doria et al 2008 reported that pregnant women with SLE are at high risk of developing lupus flare, a tendency to occur in the third trimester. ${ }^{2,3}$

Vinet et al 2012 reported that mothers with SLE had fewer live births than normal women. Clowse et al 2007 reported that pregnancy complications often occur in mothers with SLE, namely premature birth and pre- 
eclampsia. Andrade et al 2008 reported that the abortion rate in SLE patients was closely related to comorbid disease and SLE disease activity before pregnancy. 4

Anti-Ro/SSA and anti-La/SSB are one type of antinuclear antibody (ANA) in SLE. Satoh M et al 2012 reported that Anti-Ro/SSA was the most common antibody found in positive ANA, anti-Ro/SSA was found $35 \%$ while anti-La/SSB was found 15\%, more common in women than men with a ratio $3: 1$. Neiman et al 2000 reported a pregnancy with SLE that had positive anti-Ro/SSA, the risk for NLE was 6-13\% and could reach up to $25 \%$ if the mother had previously given birth to a child with NLE, CHB may occur in 15$20 \%$ and cutaneous lupus may occur in 6\%. Eronen et al 2000 reported an incidence of NLE with CHB of 50$60 \%$, while that of NLE with cutaneous lupus was 25$30 \%$ and NLE with CHB and cutaneous lupus was 4$10 \% .2,5$

Based on the description above, we are interested to write a review of the management of systemic lupus erythematosus with anti SSA and SSB positive pregnancies. Early diagnosis and therapy can prevent complications in neonates with mothers who have positive anti-Ro/SSA and anti-La/SSB.

\section{Systemic lupus erythematosus with anti SSA and SSB positive pregnancies}

Pregnancy can affect the manifestation of SLE. The increase in intravascular volume in pregnancy can worsen already abnormal cardiac and renal function.
An increase in the glomerular filtration rate by $50 \%$ during pregnancy may exacerbate previously stable proteinuria. Pregnancy-induced hypercoagulability increases the risk of thrombosis associated with rheumatic and musculoskeletal disease (RMD). Calcium requirements for fetal bone development and breastfeeding can exacerbate maternal osteoporosis. In addition, normal pregnancy symptoms such as malar erythema, chloasma gravidarum, anemia, increased erythrocyte sedimentation, and diffuse arthralgia will be similar to the symptoms of active RMD. Pregnancyinduced hypertension syndrome (pre-eclampsia) will be similar to lupus nephritis. Hemolysis, Elevated liver enzyme levels, and low platelet levels (HELLP) syndrome or eclampsia can mimic severe SLE. Differentiating these syndromes requires the expertise of a working rheumatologist and obstetriciangynecologist. 6

Anti-Ro/SSA and anti-La/SSB antibodies are strongly associated with $\mathrm{CHB}$ and cutaneous lupus, but are rare. Congenital heart block is influenced by three main factors, firstly the maternal component with positive anti-Ro/SSA or anti-La/SSB, if the levels are not sufficient, apoptosis will not occur which will form scar tissue. The two components of the fetus that can increase the occurrence of apoptosis and fibrosis are closely related to genetics, while the third is environmental factors, namely environmental stress (intrauterine), especially hypoxia and fetal position in the uterus (Figure 1). ${ }^{7}$

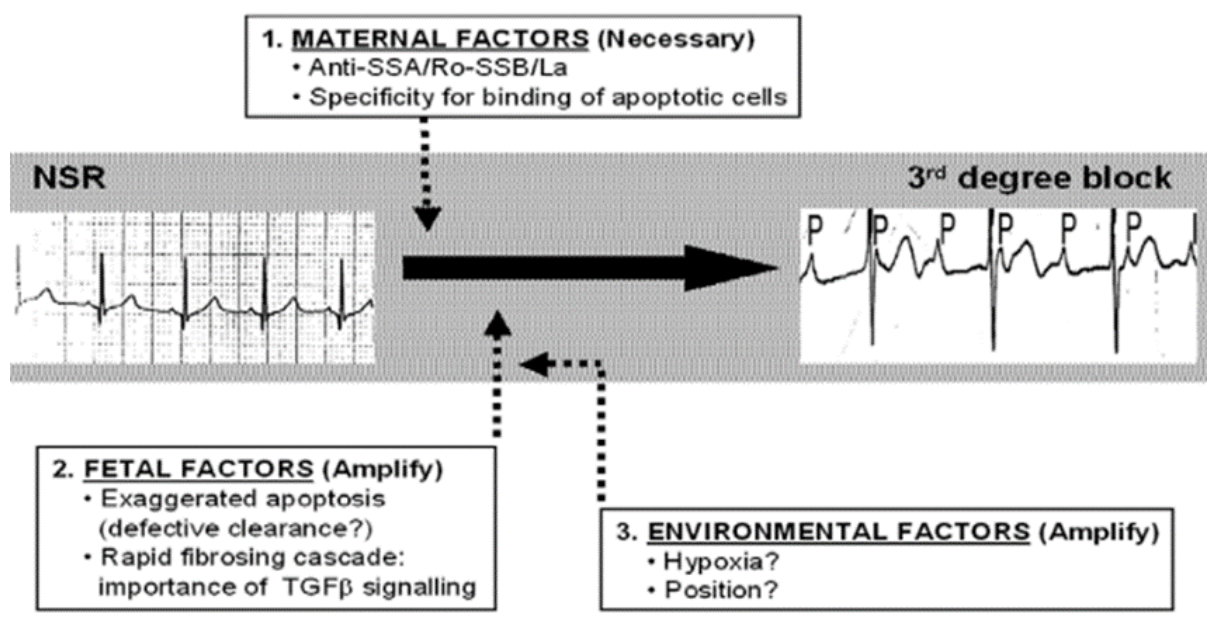

Figure 1. Factors influencing the occurrence of CHB. ${ }^{7}$ 
The molecular mechanism of anti-Ro/SSA and antiLa/SSB antibodies that can affect fetal skin and heart is still a topic of ongoing research. The presence of antiRo antibodies is necessary, but not sufficient to cause CHB. Neonatal lupus disease is influenced by interactions between maternal transplacental antibody pathways, beginning in the late first or early second trimester. This interaction may explain why this complication is still rare, because the presence of high antibody titers alone is not sufficient to cause CHB. There are two theories of the pathogenesis of CHB, namely the apoptosis hypothesis and the Ca Channel hypothesis. ${ }^{8}$

The apoptosis hypothesis explains that antiRo/SSA antibodies bind to cell surface antigens undergoing apoptosis in the remodeling process. Supposedly, the Ro antigen is intracellular, but the process of apoptosis induces translocation of antibodies to the surface of fetal cardiomyocytes. Under normal conditions, cardiomyocytes undergo apoptosis but immune complexes interfere with the clearance of these cells so that apoptotic cells induce macrophage infiltration, activation and release of cytokines such as TNF- and TGF- $\beta$. TGF- $\beta$ stimulates the differentiation of fibroblasts into myofibroblasts, leading to scar formation. Thus, fetal cardiac conduction tissue is exposed to an autoimmune reaction that causes inflammation and fibrosis, leading to irreversible CHB (Figure 2). ${ }^{8}$

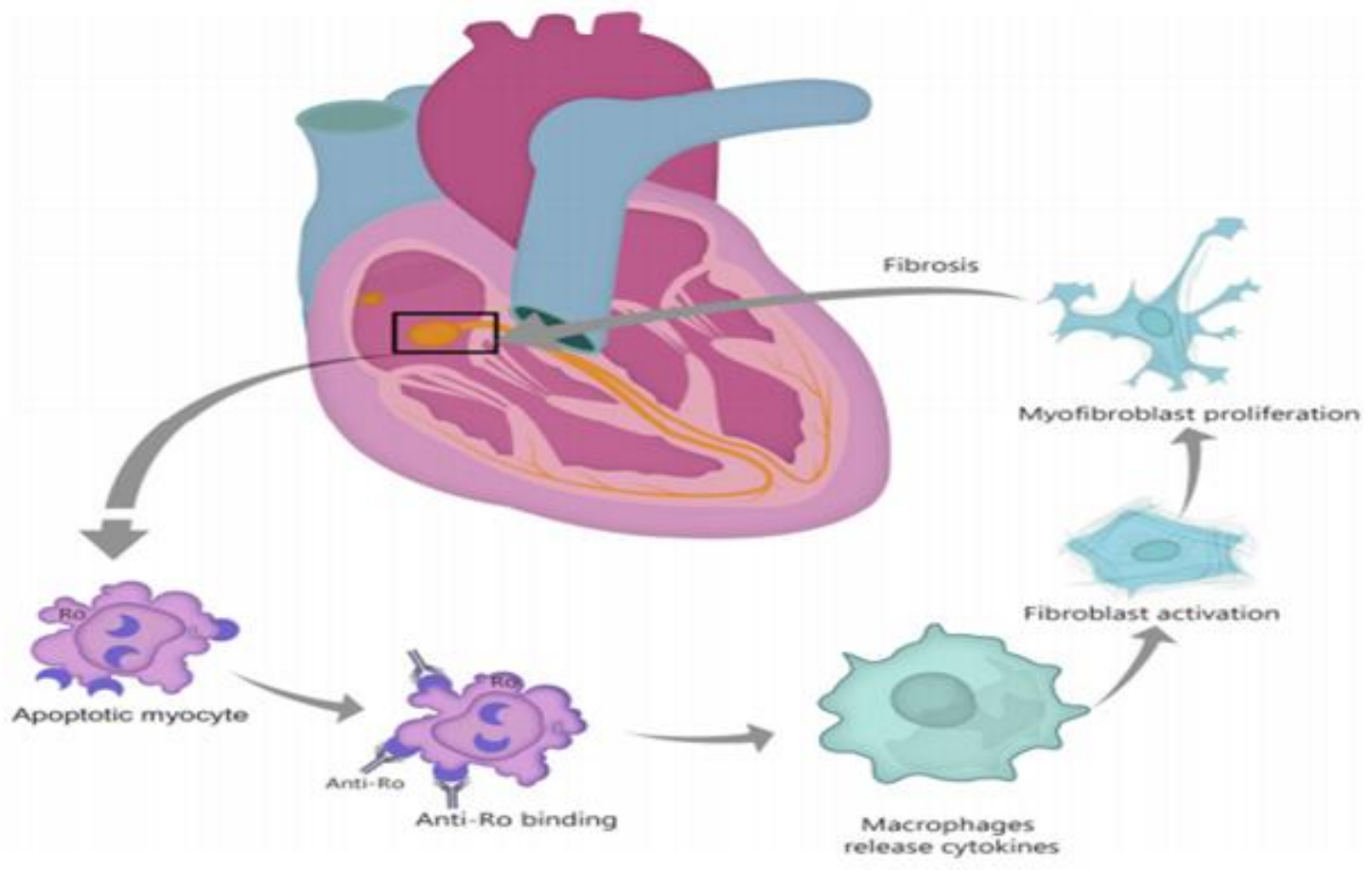

Figure 2. Apotosis hypothesis. ${ }^{8}$

Ca Channel hypothesis theory that anti-Ro antibodies inhibit calcium channels directly. AntiRo/SSA antibodies can inhibit L-type and T-type calcium channels. As a result, the short-term effect is a decrease in calcium flow, and the long-term effect is the internalization of calcium channels. This situation causes a disruption of intracellular calcium, which can lead to apoptosis, inflammation and fibrosis (Figure 3). ${ }^{8}$ 


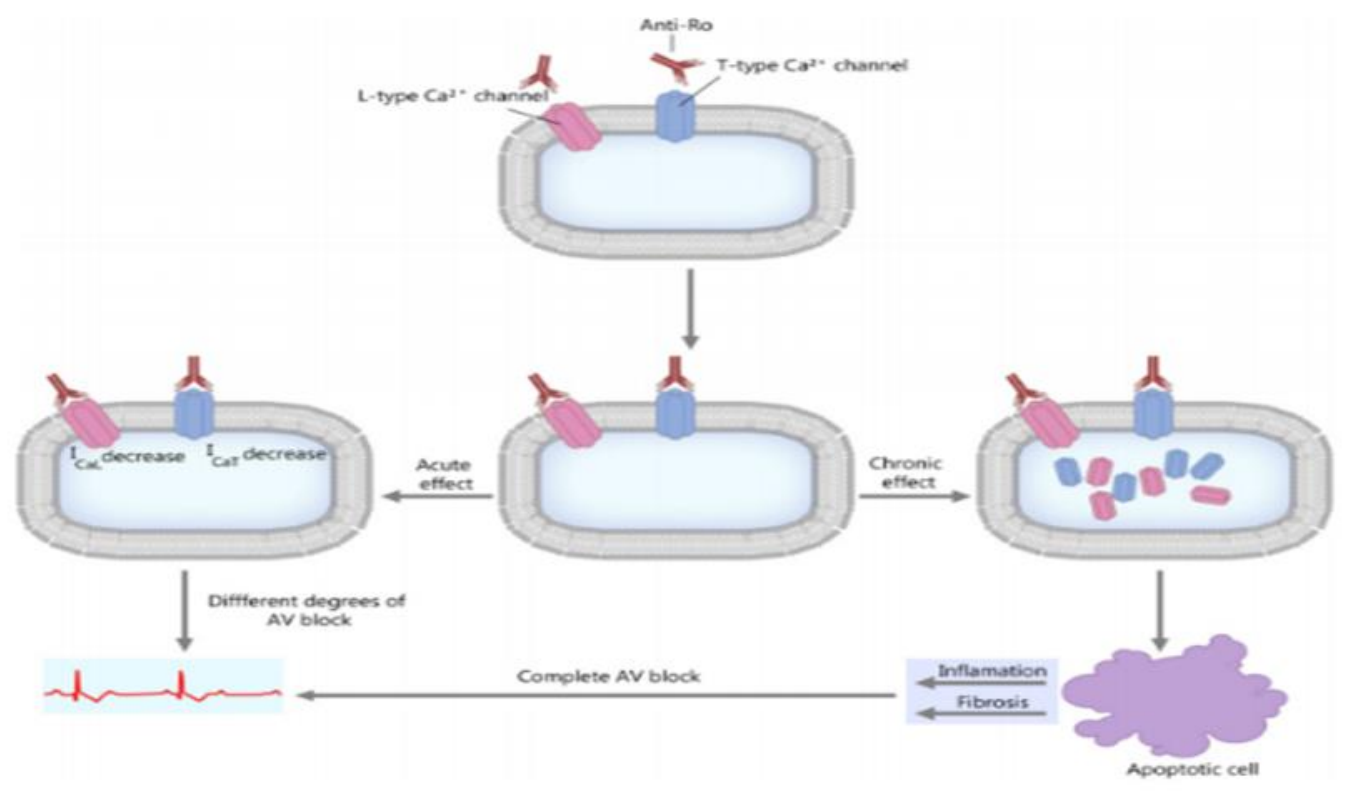

Figure 3. Calcium channel theory. ${ }^{8}$

Amanda et al 2015 reported that anti-Ro or anti-La antibodies began to cross the placenta and reach fetal tissues at 12 weeks of gestation. These antibodies can cause myocarditis, bind to apoptotic cells and cause arrhythmogenesis. Immune-mediated damage to the cardiac conduction system ends in fibrotic tissue. Grade III AV block is the most severe manifestation of neonatal lupus, because it is irreversible and has a high morbidity and mortality rate. ${ }^{9,10}$

Congenital heart block occurs at 16 to 26 weeks' gestation and is usually preceded by a lesser degree of conduction delay. However, conduction abnormalities can progress very rapidly and $\mathrm{CHB}$ is often the first rhythm abnormality detected. Rein et al 2009 reported that all exposed fetuses should be monitored weekly between 16 and 26 weeks of gestation and subsequently evaluated every two weeks. Early detection of conduction abnormalities such as a long PR interval should be considered a danger signal. Lun Hon et al 2012 reported that the most serious complication in neonates was $\mathrm{CHB}$, requiring a pacemaker, Izmirly et al 2010 reported that 10\% would progress to cardiomyopathy after birth despite a pacemaker, and the 10 -year mortality rate was 20$35 \% .^{3,11}$

\section{Therapy of systemic lupus erythematosus with anti SSA and SSB positive pregnancies}

In women with lupus who are currently pregnant with active SLE disease, requiring medical therapy, ACR recommends steroid-sparing therapy in accordance with pregnancy, because high doses of glucocorticoid therapy continuously have the potential to harm the mother and fetus. SLE patients with antiphospholipid syndrome, the ACR recommends therapy with aspirin 81-100 mg daily for prophylaxis of the risk of pre-eclampsia. Pregnancy with anti-Ro/SSA or anti-La/SSB antibodies but no history of infant with $\mathrm{CHB}$ or NLE, the ACR recommends serial fetal echocardiography at unspecified intervals (less than 1 week) starting between 16 and 18 weeks and continuing until week 26. For women with infants with a history of CHB or NLE, the ACR recommends weekly fetal echocardiography, starting at weeks 16-18 and continuing through week 26 (Figure 4). ${ }^{6}$

In anti-Ro/SSA and/or anti-La/SSB pregnancies with known grade I and II CHB on echocardiography, treatment with oral dexamethasone $4 \mathrm{mg}$ daily is recommended because fluorinated glucocorticoids such as dexamethasone and betamethasone can cross the placenta. low to moderate, but nonfluorinated glucocorticoids such as prednisone and prednisolone are largely metabolized before reaching the fetus. ${ }^{6}$

American College of Rheumatology and European League Against Rheumatism (EULAR) 2019 in antiRo/SSA and/or anti-La/SSB positive patients are 
recommended to administer hydroxychloroquine before pregnancy or at least at less than 16 weeks of age to prevent neonatal lupus. Consumption of hydroxychloroquine is continued throughout pregnancy. The use of hydroxychloroquine during pregnancy in patients with SLE can reduce the number of flares and hypertension. ${ }^{12}$

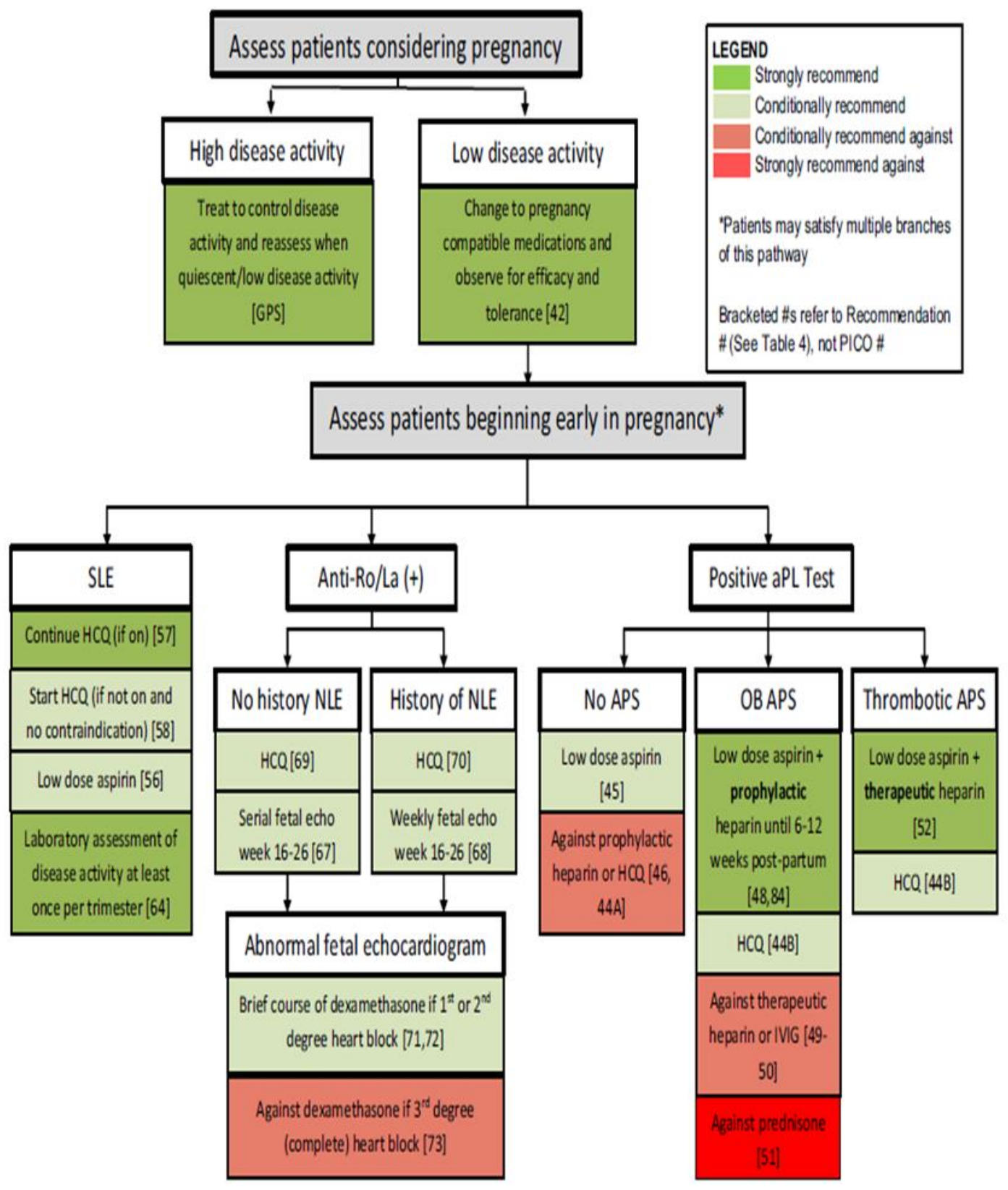

Figure 4. Pregnancy management in rheumatic and musculoskeletal disease. ${ }^{6}$

Non-steroidal anti-inflammatory drugs (NSAIDs) can be given for the treatment of arthralgia or serositis at the lowest possible dose for a short period of time and are recommended to be discontinued completely after the 32 nd week of gestation. After this time, there is a high risk of fetal and maternal hemorrhage, and fetal renal dysfunction, oligohydramnios, and premature closure of the ductus arteriosus may occur. The use of some drugs methotrexate, cyclophosphamide and mycophenolate should be discontinued for 3 months before planning a pregnancy. ${ }^{12,13}$ 
Ostensen M et al 2006 reported azathioprine is one of the recommended immunosuppressive safety in pregnancy, with a maximum dose of $2 \mathrm{mg} / \mathrm{kg} /$ day, to avoid the risk of cytopenia and suppression of the immune system in the fetus and is also recommended for severe maternal conditions or refractory to use of corticosteroids. Lockshin M, et al 2008 reported that other immunosuppressive drugs that are safe for pregnancy and there have been no reports of risks to the fetus are calcineurin inhibitors, for example tacrolimus and cyclosporine. Leflunomide was considered teratogenic and was discontinued for 2 years or a washing procedure was performed prior to conception. Research on rituximab or belimumab is very limited, we suggest discontinuing it before conception. ${ }^{14}$

Cyclophosphamide, mycophenolate mofetil, leflunamide, and methotrexate are teratogenic and should not be used during pregnancy. Mycophenolate mofetil should be discontinued at least 6 months before conception and converted to azathioprine, for the small risk of flares during the period of pregnancy. Aspirin 75 $\mathrm{mg} /$ day from 12 weeks of age should be given during pregnancy and is associated with a $10 \%$ reduction in the risk of pre-eclampsia, premature birth at less than 34 weeks of age, perinatal mortality, low birth weight, and maternal death. If there is a severe and lifethreatening recurrence during pregnancy, high-dose pulsed methylprednisolone can be considered according to the degree of disease activity taking into account the benefits and risks. ${ }^{12,14}$

\section{Conclusion}

Pregnancy in SLE patients with positive antiRo/SSA and/or anti-La/SSB positive fetal echocardiography was performed from 16 weeks to 26 weeks of gestation. There is no effective therapy if $\mathrm{CHB}$ was involved. Oral dexamethasone at a dose of $4 \mathrm{mg}$ daily is recommended to reduce the incidence of $\mathrm{CHB}$ in neonates, but this therapy cannot be given in the setting of third-degree heart block. The use of hydroxychloroquine during pregnancy in patients with SLE is recommended to reduce the incidence of flares and hypertension.

\section{References}

1. Phansenee S, Sekararithi R, Jatavan P, Tongsong T. Pregnancy outcomes among women with systemic lupus erythematosus: a retrospective cohort study from thailand. Lupus. 2017; 27(1): 158-64.

2. George S, Michelle P. Epidemiology of systemic lupus erythematosus: an update. Curr Opin Rheumatol. 2018; 30(2): 144-50.

3. Guilherma RJ, Claudia MP, Nilson, RJ, Flavia CS, Evandro MK, Mario GC, et al. Understanding and managing pregnancy in patients with lupus. Autoimmune Disease. 2015; 15: 1-18.

4. Caroline LK, Catherine NP. Management of systemic lupus erythematosus during pregnancy: challenges and solutions. Open Access Rheumatology. 2017; 9: 37-53.

5. Nuria M, Sergio P, Angel R, Francisco A, Maria $\mathrm{MC}$, Edurne $\mathrm{HZ}$ et al. Obstetric and perinatal outcome in anti -Ro/SSA-positive pregnant women: a prospective cohort study. Immunol Res. 2017; 65: 487-94.

6. Lisa R S, Bonnie LB, Eliza EC, Christina C, Megan EB, Wendy M, et al. 2020 American College of Rheumatology guideline for the management of reproductive health in rheumatic and musculoskeletal diseases. Arthritis \& rheumatology. 2019; 72(4): 529-56.

7. Buyon JP, Clancy RM. Antibodies to SSA/Ro and SSB/La: potential mechanisms of tissue injury in neonatal lupus-congenital heart block. Systemic Lupus Erythematosus. 2007; 1: 248-56.

8. Mihaela RP, Andreea D, Ciprian J, Anca MC, Anamaria Z, Ancamaria P. A broader perspective on anti-Ro antibodies and their fetal consequences- a case report and literature review. Diagnostics. 2020; 10(7): 1-26.

9. Amanda SKOG, Linda L, Peter C, Marie WH, Sven ES. Outcome in 212 anti-Ro/SSA-positive pregnancies and population based incidence of congenital heart block. AOGS. 2015; 95: 98-105.

10. Kam LH, Leung AKC. Neonatal lupus erythematosus. Autoimmune Disease. 2012; 2: 16. 
11. Brucato A, Doria A, Castellino G, Franchesini F, Faden D, Pisono MP, et al. Pregnancy outcome in 100 women with autoimmune disease and antiRo/SSA antibodies: a prospective controlled study. Lupus. 2002; 11: 716-21.

12. Sumariyono, Kalim H, Setyohadi B, Hidayat R, Najirman, Hamijoyo, et al. Diagnosis dan pengelolaan lupus eritematosus sistemik. Perhimpunan Reumatologi Indonesia. 2019

13. Antonio B, Rolando C, Roberto C, Veronique R, Jill B. Pregnancy outcomes in patients with autoimmune disease and Anti-Ro/SSA antibodies. Clinic Rev Allerg Immunol. 2011; 40: 27-41.

14. Aisha L, Michelle P. Managing lupus patients during pregnancy. Best Pract Res Clin Rheumatol. 2013; 27(3): 1-8. 